\title{
Improved efficiency for respiratory motion compensation in three-dimensional flow measurements
}

\author{
Mehmet Akcakaya ${ }^{1 *}$, Praveen Gulaka ${ }^{3}$, Tamer A Basha', Thomas H Hauser ${ }^{1}$, Warren J Manning ${ }^{1,2}$, Reza Nezafat ${ }^{1}$ \\ From 16th Annual SCMR Scientific Sessions \\ San Francisco, CA, USA. 31 January - 3 February 2013
}

\section{Background}

Phase contrast (PC) CMR is clinically used for in-vivo assessment of blood flow in cardiovascular disease [1]. Typically, a through-plane 2D acquisition is performed for evaluating the blood flow. Recently, 3D time-resolved PC CMR has been used for quantification and visualization of the blood flow in all three directions of a volume [2]. However, such acquisitions require long scan times, which are further prolonged by the need for respiratory motion compensation, typically using respiratory navigators. In this study, we hypothesized that respiratory gating the center of k-space only will yield similar measurements to a fully respiratory-gated acquisition, since the phase information is mainly coming from the central k-space, and evaluated these two gating approaches.

\section{Methods}

12 subjects were recruited $(33.2 \pm 15.8$ years; 5 males) for 3D flow CMR on a 1.5T Philips Achieva magnet. Images were acquired axially using a GRE sequence $\left(\mathrm{TR} / \mathrm{TE} / \alpha=5.7 / 3.4 \mathrm{~ms} / 10^{\circ}\right.$, resolution $=2 \times 2 \times 4 \mathrm{~mm}^{3}$, FOV $=340 \times 340 \times 40 \mathrm{~mm}^{3}$ ) in a volume covering the ascending and descending aorta, and the aortic bifurcation. Only foot-head flow encoding was used to provide an adequate temporal resolution of $23.2 \mathrm{~ms}$ for the measurements. The nominal scan time for this scan was 12 minutes at $60 \mathrm{bpm}$.

For respiratory motion compensation, two gating \& tracking strategies were used with a $7 \mathrm{~mm}$ gating window: 1) All of k-space is acquired within the gating window (fully-gated), 2) Central k-space (corresponding to $4 \%$ of

'Medicine, Beth Israel Deaconess Medical Center, Harvard Medical School, Boston, MA, USA

Full list of author information is available at the end of the article $\mathrm{k}$-space) is acquired within the gating window, and the rest of k-space is acquired without any gating (centergated). Two fully-gated acquisitions were performed. Acquisition time was recorded for each scan. Stroke volume measurements were performed both on the ascending and the descending aorta for all acquisitions. Bland-Altman analyses were performed to compare the flow measurements between different gating strategies (fully-vs. center-gated), as well as to compare the interscan variation for the fully-gated strategy (i.e., fully-gated $\# 1$ vs. \#2).

\section{Results}

Figures 1 and 2 show the Bland-Altman analysis for stroke volume for the ascending and descending aorta respectively. The intra-scan variability observed for fully-gated scans is equivalent to or higher than the variability observed between fully-gated and centergated strategies. Furthermore, the proposed center-gated strategy has significantly shorter acquisition time compared to the fully-gated strategy $(13: 19 \pm 3: 02$ vs. 19:35 \pm 5 :02, $\mathrm{P}<0.001)$.

\section{Conclusions}

We proposed and demonstrated a more efficient respiratory gating strategy for 3D PC CMR. No systematic variation was observed for the stroke volume (cardiac output) measurements between the proposed strategy and the fully-gated one, with the proposed strategy having a markedly shorter acquisition time.

\section{Funding}

NIH: K99HL111410-01; R01EB008743-01A2; Samsung Electronics. 


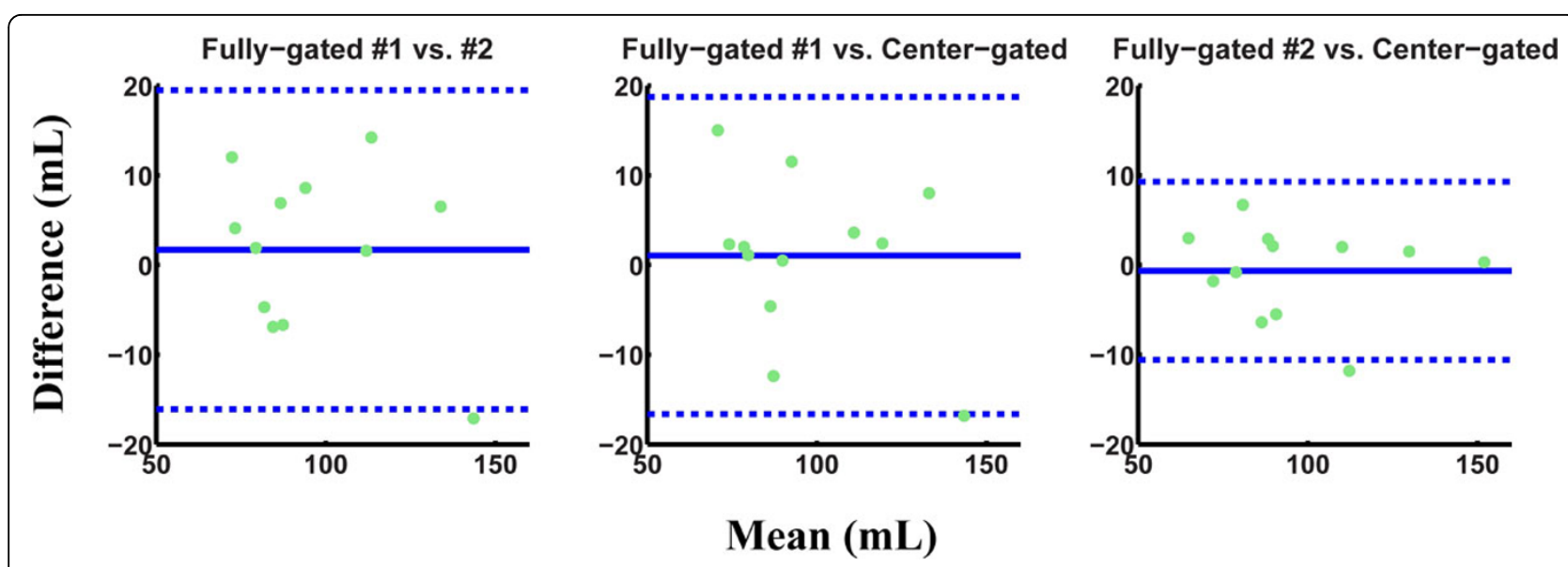

Figure 1 Bland-Altman analysis of the stroke volume through the ascending aorta for various navigator gating strategies. The center-gated scans are clinically identical to the fully-gated scans for these acquisitions.

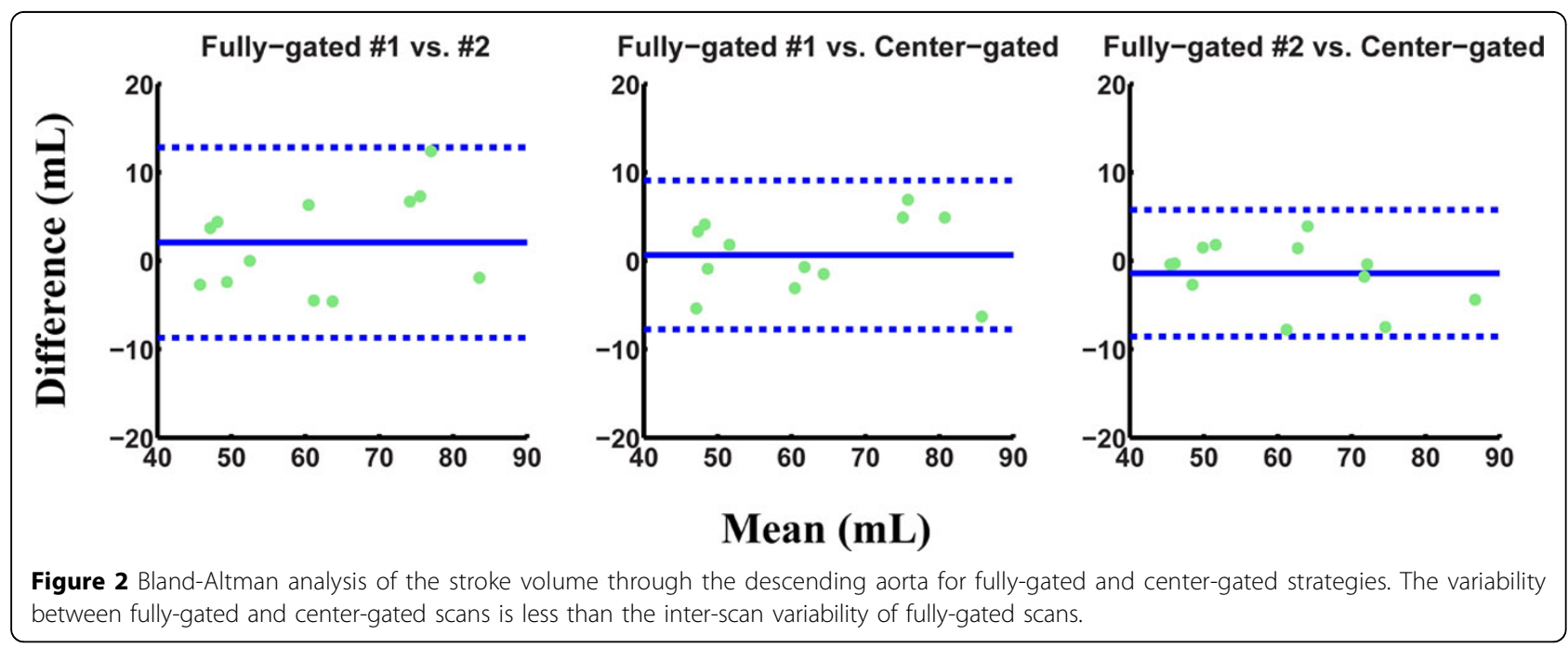

Author details

${ }^{1}$ Medicine, Beth Israel Deaconess Medical Center, Harvard Medical School, Boston, MA, USA. ${ }^{2}$ Radiology, Beth Israel Deaconess Medical Center, Harvard Medical School, Boston, MA, USA. ${ }^{3}$ Health and Medical Equipment, Samsung Electronics Co., Suwon, Republic of Korea.

Published: 30 January 2013

References

1. Markl:. JCMR 2011

2. Markl.. JMRI 2003.

\section{doi:10.1186/1532-429X-15-S1-P30}

Cite this article as: Akcakaya et al.: Improved efficiency for respiratory

motion compensation in three-dimensional flow measurements. Journal of Cardiovascular Magnetic Resonance 2013 15(Suppl 1):P30.

Submit your next manuscript to BioMed Central and take full advantage of:

- Convenient online submission

- Thorough peer review

- No space constraints or color figure charges

- Immediate publication on acceptance

- Inclusion in PubMed, CAS, Scopus and Google Scholar

- Research which is freely available for redistribution 